\title{
From reverse CART to antegrade wire access: a guide to externalisation, tip-in, rendezvous, and snaring from the APCTO club
}

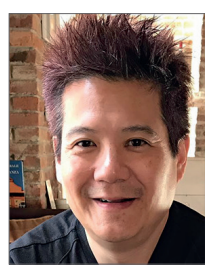

Eugene B. Wu ${ }^{1 *}$, MD, Hsien-Li Kao², MD, Sidney $\mathrm{Lo}^{3}, \mathrm{MD}$, Soo Teik Lim4, MD, Lei Ge ${ }^{5}, \mathrm{MD}$, Ji-Yan Chen 6 , MD, Jie Qian', MD, Seung-Whan Lee ${ }^{8}$, MD, PhD, Scott A. Harding 9 , MD, Etsuo Tsuchikane ${ }^{10}, \mathrm{MD}, \mathrm{PhD}$

1. Prince of Wales Hospital, Hong Kong; 2. National Taiwan University Hospital, Taipei, Taiwan; 3. Liverpool Hospital, Sydney, Australia; 4. National Heart Centre, Singapore; 5. Shanghai Zhongshan Hospital, Shanghai, China; 6. Guangdong General Hospital, Guangdong, China; 7. Beijing Fuwai Hospital, Beijing, China; 8. Asan Medical Centre, Seoul, Republic of Korea; 9. Wellington Hospital, Wellington, New Zealand; 10. Toyohashi Heart Centre, Toyohashi, Aichi, Japan

This paper also includes supplementary data published online at: www.asiaintervention.org

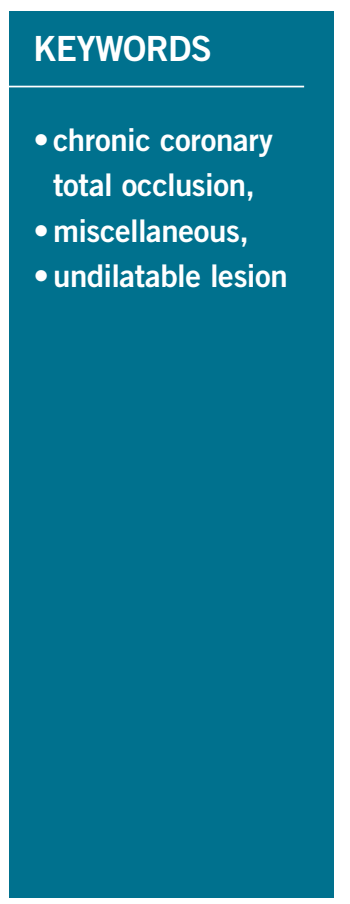

\section{Abstract}

We at the Asia Pacific Chronic Total Occlusion (APCTO) club, provide this review to address the gap between reyerse controlled antegrade and retrograde subintimal tracking (CART) and antegrade wire access. We describe the usual method for wire externalisation. We then address how to deal with failure to wire the proximal part of the chronic total occlusion (CTO) vessel or the guiding catheter. After successful antegrade guiding catheter wiring, we address the problem of failing to cross the CTO body with the retrograde microcatheter and we recommend the use of a retrograde small balloon, reversion to traditional CART, retrograde knuckle wiring into the subintimal space and antegrade scratch and go, and external cap crush. We also propose rendezvous type tip-in and describe the way to do this to overcome problems. In conclusion, we review and make recommendations for methods to gain antegrade wire access after successful reverse CART. We have addressed each failure mode in detail covering the different options, balancing risks and success rates. Our recommendations focus upon safety first and ease of use. We hope this work will help all retrograde operators to further improve the safety, efficacy, and success rates of their retrograde procedures.

*Corresponding author: Department of Medicine and Therapeutics, Prince of Wales Hospital, Ngan Shing Street, Shatin, Hong Kong. E-mail: cto.demon@gmail.com 


$\begin{array}{ll}\text { Abbreviations } \\ \text { APCTO } & \text { Asia Pacific Chronic Total Occlusion Club } \\ \text { CART } & \text { controlled antegrade and retrograde subintimal tracking } \\ \text { CTO } & \text { chronic total occlusion } \\ \text { PCI } & \text { percutaneous coronary intervention } \\ \text { GC } & \text { guiding catheter } \\ \text { MC } & \text { microcatheter } \\ \text { LAD } & \text { left anterior descending artery } \\ \text { PDA } & \text { posterior descending artery } \\ \text { RCA } & \text { right coronary artery } \\ \text { IVUS } & \text { intravascular ultrasound }\end{array}$

\section{Introduction}

The retrograde approach for CTO has adopted two major changes since its original description more than a decade ago ${ }^{1,2}$. First, the introduction of the Corsair microcatheter (Asahi Intecc, Aichi, Japan) in $2009^{3}$ transformed the cumbersome small balloon septal channel dilatation technique into a simple single microcatheter/channel dilator method for channel crossing. This led to the inevitable dominance of reverse controlled antegrade and retrograde tracking (Rev-CART) as the go-to technique for achieving CTO segment crossing. Secondly, the widespread availability of the Gaia wire series (Asahi Intecc) since 2012 improved retrograde wire control markedly, leading to the subsequent development of the more efficient "directed reverse CART" . Since then, the retrograde approach has reached a plateau in its development with widespread global adoption ${ }^{5-11}$, and a significant improvement of overall success in CTO percutaneous coronary intervention $(\mathrm{PCI})^{5,8,10-13}$. The retrograde approach had been incorporated into the hybrid algorithm ${ }^{14,15}$, and a step-by-step guide has been published ${ }^{16,17}$. Along with the landmark work of Wu et al ${ }^{18}$, most of the procedural details of retrograde CTO PCI have been described. However, a more up-to-date version is required in view of recent technical and device advancements.

Our group, the Asian Pacific Chronic Total Occlusion Club (APCTO), is composed of 10 highly experienced retrograde operators who have published recently an overall algorithm for CTO interventions ${ }^{19}$, a comprehensive retrograde algorithm ${ }^{20}$, and a state-of-the-art guide to CTO wiring ${ }^{21}$. However, we noticed that publications describing what to do after successful retrograde wire crossing are scarce. This particular gap between successful reverse CART (rev-CART) and establishment of an antegrade wire access involves a wide array of techniques that are not well described in the literature. Although we often think that successful rev-CART guarantees a successful procedure, this is not necessarily true. These infrequently used techniques are important in certain subsets of patients: those where the retrograde microcatheter cannot pass the CTO, those with tortuous proximal vessel anatomy, and rarely, for ipsilateral single guiding retrograde percutaneous coronary intervention (PCI). Familiarity with these techniques and using them safely is an important part of the armoury of the CTO interventionist. The aim of this present work, therefore, is to review and describe these techniques in comprehensive detail, providing useful expert instructions to retrograde operators worldwide.

\section{Default wire externalisation}

This is recommended in the majority of retrograde CTO cases.

\section{WIRING INTO AND ANCHORING INSIDE THE ANTEGRADE GUIDING CATHETER}

Once the retrograde wire enters the proximal true lumen following successful rev-CART, it should be further advanced into the antegrade guiding catheter (GC). A trapping balloon is then inflated inside the antegrade GC to anchor the retrograde wire. This provides strong wire support for the retrograde microcatheter (MC) to be pushed across the CTO body into the antegrade GC. Once the retrograde $\mathrm{MC}$ is securely inside the antegrade $\mathrm{GC}$, we can deflate the trapping balloon and replace the retrograde wire with an externalisation wire such as an RG3 wire (Asahi Intecc) or ViperWire ${ }^{\circledR}$ (Cardiovascular Systems Inc, St Paul MN, USA).

\section{ANCHORING THE MC}

Some operators prefer to push the trapping balloon forward and inflate it to anchor the retrograde $\mathrm{MC}$ in the antegrade $\mathrm{GC}$ before exchanging the guidewires. There are two advantages for this practice: 1) the balloon will anchor and stabilise the retrograde MC during exchange, and 2) the inflated balloon will prevent back-bleeding during disconnection of the antegrade haemostatic valve/connector while completing wire externalisation.

\section{PASSING THROUGH THE ANTEGRADE HAEMOSTATIC VALVE/ CONNECTOR}

The operator should push the externalisation wire until the wire tip is about to emerge from the antegrade GC hub, judged and estimated by the remaining wire length, under fluoroscopic guidance. Then the operator should put a wire introducer through the antegrade haemostatic valve/connector in parallel to the anchor balloon (Figure 1A), and disconnect the connector (Figure 1B). The retrograde externalisation wire should then be pushed forward out of the hub of the antegrade GC (Figure 1C), and the wire tip is brought into the tip of the wire introducer (Figure 1D). Finally, the haemostatic valve/connector should be reconnected to the GC, with both the trapping balloon and wire introducer still in place (Figure 1E). The wire introducer is then removed, and the externalisation wire may be used to deliver devices antegradely (Figure 1F). The above-described wire externalisation should be used as default whenever possible in the retrograde approach.

\section{WHEN TO CONTINUE ON EXTERNALISED WIRE AND WHEN TO SWITCH TO ANTEGRADE WIRE?}

Once we have externalised a wire, we have the choice to either work on the externalised wire or to put an MC or dual lumen catheter over the externalised wire and place a second antegrade wire. Although the default position is to use the externalised wire as 


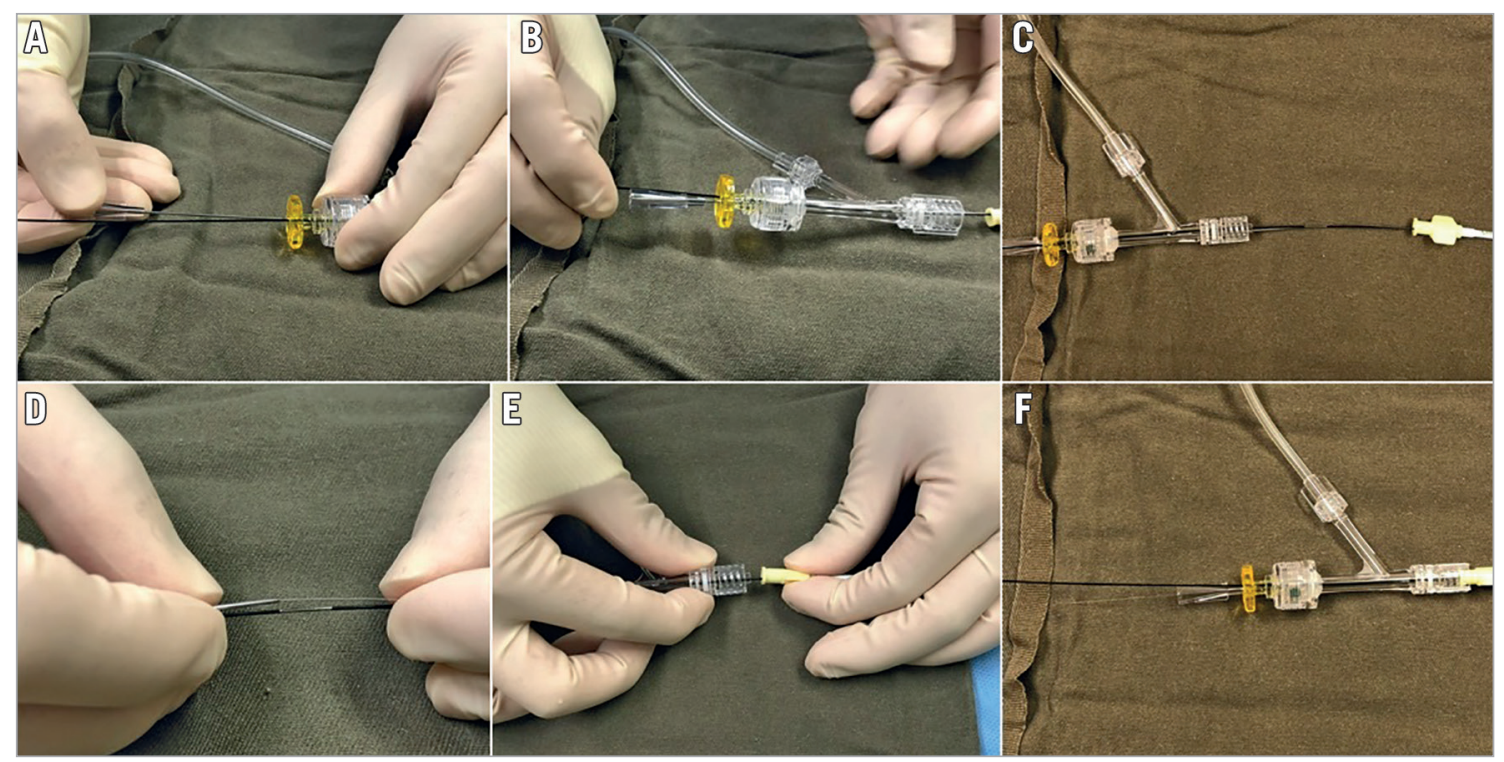

Figure 1. Passing the externalisation wire through the antegrade Y-connector. A) Insertion of wire introducer in parallel to the anchor balloon shaft through the antegrade Y-connector. B) Disconnected Y-connector is now pulled back over the anchor balloon. C) Retrograde wire is pushed out of the antegrade guiding hub. D) Putting the externalisation wire into the wire introducer. E) Y-connector is brought near to guiding hub for reconnection. F) Removal of introduced wire to allow passage of devices.

a working wire as it provides better support for tracking devices, in some cases the option of switching to antegrade wire is preferable.

If we need to stent beyond where the retrograde channel enters the CTO vessel due to distal disease, then it is preferable to switch to antegrade wire. This usually occurs in the left anterior descending artery (LAD) where the posterior descending artery (PDA) septal channel often enters the mid-LAD with significant disease in the distal LAD that requires stenting. In this case, using a dual lumen catheter to deliver a second wire into the septal channel and then pulling back this wire to wire the true LAD is the best method. We must remove the externalised wire before stenting to avoid wire trapping. Other reasons to switch to antegrade wire include the retrograde channel entering near the distal CTO cap or the need to protect a side branch distal to the retrograde channel entry site.

However, sometimes, this default method of wire externalisation fails and we now turn to addressing these problems.

\section{Failure to access proximal vessel lumen with retrograde wire}

The operator may fail to manipulate a retrograde wire through the proximal vessel lumen despite successful rev-CART achieving proximal vessel true lumen position of the retrograde wire. This is almost always due to migration of the retrograde wire tip back into the intraplaque or subintimal space in the proximal vessel. It occurs more frequently when the proximal vessel is significantly diseased and tortuous, when balloon angioplasty had been performed in the proximal vessel segment, and when a high penetration force retrograde wire is used for rev-CART.
In this scenario, we recommend the use of a guide extension, as described by Mozid et $\mathrm{al}^{22}$. A guide extension catheter such as GuideLiner ${ }^{\circledR}$ (Teleflex Medical; Wayne, PA, USA) or GUIDEZILLA $^{\mathrm{TM}}$ (Boston Scientific Corp; Marlborough, MA, USA) should be advanced into the proximal vessel to the point where the retrograde wire is in the true lumen, and the retrograde wire may be manipulated easily into the guide extension. A 2.5 or $3.0 \mathrm{~mm}$ balloon inflation is sometimes needed to dilate the proximal vessel and facilitate guide extension catheter delivery. The use of a guide extension catheter is almost always successful in these cases.

\section{Failure to manipulate the retrograde wire into the antegrade GC TIPS AND TRICKS FOR WIRING INTO THE ANTEGRADE GC}

There are certain tricks to increase the efficiency of wiring into the antegrade GC with the retrograde wire. We have to recognise that manipulation of the antegrade $\mathrm{GC}$ is far more important than manipulation of the retrograde wire. The operator should ensure that the antegrade GC is coaxial with the coronary artery. For example, in right coronary artery (RCA) CTO, often the antegrade GC is pointing towards the anterior wall of the right coronary ostium. Therefore, applying clockwise rotation to the GC in the right anterior oblique view will enhance its coaxiality with the right coronary ostium and facilitate retrograde wring into the antegrade GC. The operator should push the retrograde wire forward and observe its trajectory, and then manipulate the antegrade GC to cover the expected area that the retrograde wire will travel to (Moving image 1, Moving image 2). Simultaneous manipulation of the retrograde wire and the 
antegrade GC may also be very helpful. However, certain unfavourable anatomies may require the use of a guide extension catheter. Inserting a guide extension catheter into the proximal part of the CTO vessel makes wiring into the antegrade system much easier, since it will always be coaxial to the vessel course.

\section{CHANGE THE RETROGRADE WIRE}

If the operator failed in all the above methods to wire into the antegrade GC, it is usually due to the difficulty of controlling a stiff high penetration force retrograde wire. We recommend the operator to switch the retrograde wire to a more controllable, slippery soft wire, such as $\mathrm{SION}^{\circledR}$ black (Asahi Intecc). To do this, you need to push the retrograde MC across the CTO body into the proximal true lumen of the CTO vessel. The first step is to push the retrograde guidewire as far up the aorta as possible (Figure 2A), ideally passing the arch into the descending aorta. The bend at the arch will provide extra support for this wire and allow us to track the MC across. If this fails, consider using an antegrade balloon to trap and anchor the retrograde wire inside the proximal part of the CTO vessel (Figure 2B)

If this too fails, exchange the retrograde $\mathrm{MC}$ for a new low profile rotational channel dilator type $\mathrm{MC}$, such as the Turnpike ${ }^{\circledR}$ LP (Teleflex) or Corsair Pro XS (Asahi Intecc), which will often be able to cross the CTO body (Figure $2 \mathbf{C}$ ). Once the retrograde MC has passed through the CTO body, you can exchange the retrograde high penetration force wire for a controllable soft wire to wire the antegrade GC or guide extension catheter (Figure 2D) and complete the externalisation (Figure 2E) and stenting (Figure 2F).
When all the above techniques are used properly in a stepwise fashion, the antegrade GC wiring will be successful in the majority of retrograde CTOs. However, there are still occasional cases where retrograde wire snaring, as originally described by Otsuka et $\mathrm{al}^{23}$, is required.

\section{OUR POSITION ON RETROGRADE WIRE SNARING}

We regard snaring of the retrograde wire as a last resort, to be considered only after all other methods have been exhausted. We emphasise the small but inherently real risk associated with snaring, as Fang et $\mathrm{al}^{24}$ have pointed out. These issues include: 1) the danger of failure to release the snared retrograde wire inside the antegrade GC, and 2) failure to remove the snared retrograde wire from the retrograde $\mathrm{MC}$ due to an excessive bend created by the snaring. Although previous authors have suggested several methods to rectify these situations, these methods are not always reliable ${ }^{25}$. There is also a small but unavoidable risk of stroke, with possible plaque embolism during snare manipulation in the aorta. The APCTO club thus recommends the following "safety first method" of retrograde wire snaring.

\section{SAFETY FIRST METHOD OF RETROGRADE WIRE SNARING}

When the operator decides to start retrograde wire snaring, the retrograde wire should already be in the descending aorta, as a consequence of the prior attempt to push the retrograde $\mathrm{MC}$ across CTO body for wire switch to a soft controllable wire. The 3-lobed EN Snare ${ }^{\circledR}$ (Merit Medical System, South Jordan, UT, USA) is the easiest to use to catch the wire but we recommend using a "homemade" snare with guiding extension catheter

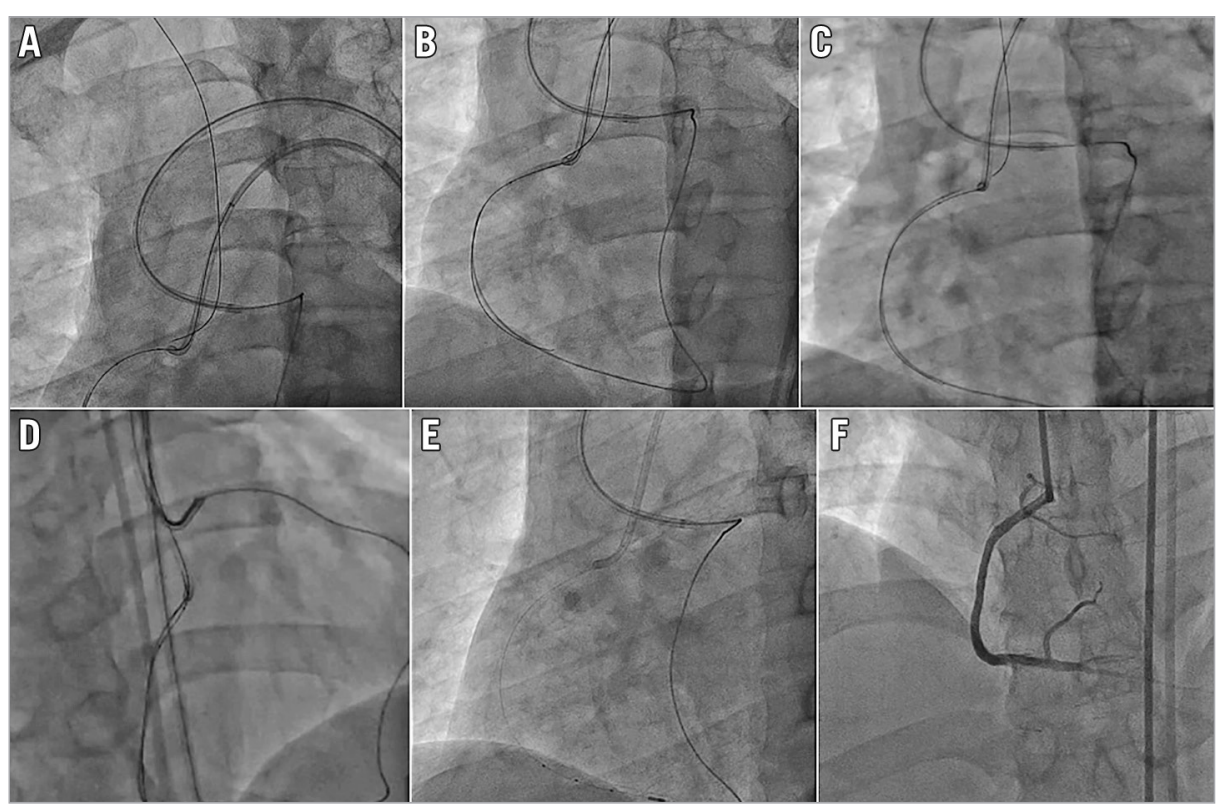

Figure 2. Initial steps to deal with failing to wire the antegrade guiding catheter. A) Pushing the retrograde wire far into the aorta. B) Using a proximal vessel anchor balloon. C) Switching to new low profile rotational retrograde MC. D) Switching to a soft wire to wire the GC. E) Successful externalisation. F) Good final results. 
and monorail balloon ${ }^{26}$. See "How to create a homemade snare": https://www.youtube.com/watch?v=wJ0if-5OEfM. The advantage of a homemade snare is that the size of the snare loop can be easily increased to as large as needed ${ }^{26}$ by pushing the wire forward (Figure $3 \mathrm{~A}$ ), and also when the snare has pulled the retrograde wire back into the guiding catheter (Figure 3B), release of the retrograde wire can be easily done by simply deflating the balloon. Since there is no connected loop once the balloon is deflated, the retrograde wire can almost always be released. Then an anchoring balloon can be placed to anchor the retrograde wire inside the antegrade $\mathrm{GC}$ allowing the retrograde $\mathrm{MC}$ to pass (Figure $\mathbf{3 C}$ ). We should aim to snare the floppy tip of the retrograde wire in the descending aorta or the arch. Snaring in the ascending aorta closer to the coronary ostium may increase the embolic stroke risk. After catching the retrograde wire, if we are using a more robust snare such as EN snare, we should first try pushing the retrograde MC across the CTO body into the aorta while pulling on the snare as anchor. Then the snared retrograde wire is released in the descending aorta and exchanged with a new soft controllable wire to wire into the guiding GC again. This will remove the risk of locking the snare with the trapped retrograde wire inside the antegrade GC.

If we cannot push the retrograde MC across the CTO body despite snare anchoring, or if we are using a homemade snare, we may try pulling the snared retrograde wire into the antegrade GC. The retrograde $\mathrm{MC}$ is then tracked into the GC before the snare is released, and then the retrograde wire may be exchanged to an externalisation wire. The risk of failing to release a soft hydrophilic wire from a snare is considered low, but still possible. If we cannot release the $190 \mathrm{~cm}$ retrograde wire from the snare, we should push the snare and wire back into the aorta and try releasing again. We can also use a balloon inside the antegrade GC to anchor the wire tip, facilitating the release of the snare. We should never, however, try to pull the whole $190 \mathrm{~cm}$ wire through the retrograde channel into the antegrade GC. This is because the proximal end of the wire is very stiff and uncoated, and together with the MC, with which it forms a single unit, may get stuck in a tortuous retrograde channel.
The alternative is to exchange the retrograde wire to an RG3 wire, and start snaring its tip at the level of the contralateral common iliac artery. This reduces the risk of embolism, and snaring in a smaller vessel such as the iliac is often easier. If we fail to release the snare on the RG3, we can always pull its snared distal tip out from the contralateral access. With its $300 \mathrm{~cm}$ exchange length, the proximal end will still be controlled outside of the retrograde GC. We do not, however, recommend wiring through the coronary with an RG3, as its $3 \mathrm{~g}$ tip load and poor torquability can easily create dissections in a diseased proximal vessel.

\section{Rendezvous techniques THE RENDEZVOUS TECHNIQUE}

Rendezvous technique refers to using an antegrade or retrograde wire to wire into, or "to meet" (in French "rendezvous"), the opposite MC. Early descriptions of this technique ${ }^{27,28}$ discuss wiring the antegrade wire into the retrograde MC. Tip-in is also a kind of rendezvous technique. In tip-in, the retrograde wire is wired into an antegrade $\mathrm{MC}$, either in the $\mathrm{GC}^{29}$ or in the coronary artery ${ }^{30}$. These techniques are used less frequently nowadays, with the availability of low profile MCs and algorithmic intravascular ultrasound (IVUS) usage in rev-CART.

The advantages of the rendezvous technique are : 1) rapid establishment of an antegrade wire track ${ }^{29}$ without the need for externalisation wire, and 2) to bail out the situation when retrograde MC fail to cross the CTO body ${ }^{30}$.

\section{THE ROLE OF RENDEZVOUS TECHNIQUES IN CONTEMPORARY RETROGRADE CTO PCI}

Many of the issues requiring original rendezvous techniques ${ }^{27-30}$ are no longer relevant, with the application of IVUS-guided revCART, low profile MCs and RG3 or Viper wires, and guide extension catheters. However, there are a few conditions that may still mandate rendezvous techniques in the current era of retrograde CTO PCI: 1) failure to cross the CTO body with the retrograde $\mathrm{MC}, 2)$ inadequate length of retrograde $\mathrm{MC}$ to reach the antegrade system, 3) the need to quickly establish an antegrade track, and 4) a single guiding catheter ipsilateral channel retrograde CTO PCI.
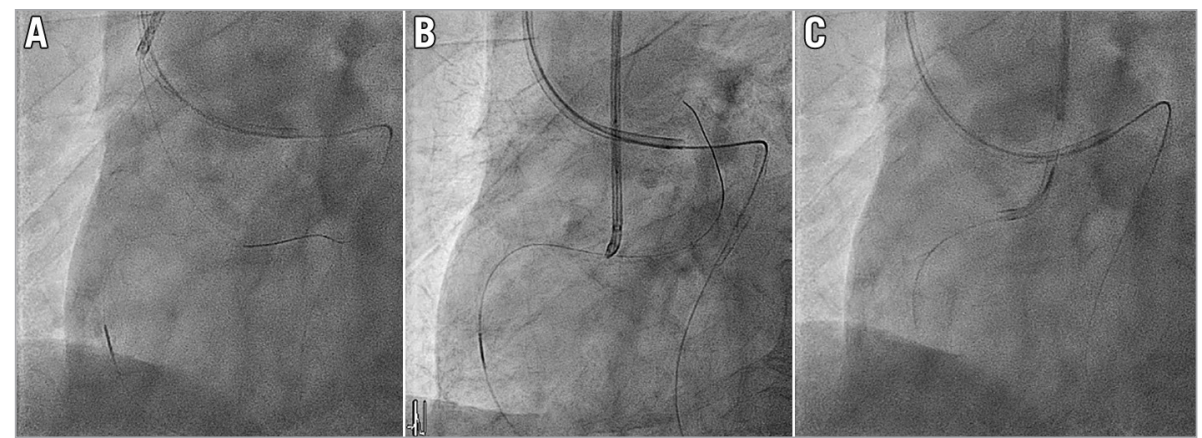

Figure 3. Homemade snare. A) Homemade snare pushed to large size. B) Snared retrograde wire. C) Anchored retrograde wire, MC passed. 


\section{FAILURE TO CROSS THE CTO WITH A RETROGRADE MC}

Despite the trapping balloon anchoring the retrograde wire in the antegrade GC, we may still fail to push the retrograde MC across the CTO body. Rendezvous techniques may be helpful, but alternative solutions may also include: 1) balloon dilatation of the CTO from the retrograde direction, 2) reverting to traditional CART ${ }^{20}$, 3) antegrade "external cap crush" by inflating a balloon in the subintimal space parallel to the CTO segment to weaken $i^{31}$, and 4) re-crossing the CTO body in a different subintimal path using retrograde knuckle wiring. These alternatives are time consuming and cumbersome, therefore in the face of these cumbersome options, rendezvous techniques remain a valid choice.

\section{FAILURE TO REACH THE ANTEGRADE SYSTEM}

With the provision of short ( 85 or $90 \mathrm{~cm}$ ) GC and guide extension catheters, failure of the retrograde MC to reach the antegrade system due to a length issue is rare. However, sometimes the final retrograde channel used may be different and significantly longer than the pre-procedural planning and we may need a rendezvous technique. Extensive calcification and tortuosity of the proximal vessel segment may also prevent antegrade guide extension catheter delivery and an unexpectedly lengthy vascular route, especially in tall patients, may prohibit a short GC from reaching the coronary ostium. The rendezvous technique is a solution in these scenarios.

\section{NEED TO RAPIDLY ESTABLISH AN ANTEGRADE TRACK}

When a dominant collateral channel is used for the retrograde approach, significant ischaemia and haemodynamic instability may occur. Proper antegrade preparation before collateral channel tracking and expedited rev-CART are helpful in this case. The rendezvous technique facilitates rapid establishment of an antegrade wire track, which minimises the ischaemia induced by the retrograde devices occupying the collateral channel.

Donor artery complications such as thrombosis and channel perforations may also interrupt our retrograde CTO PCI. Rapid establishment of an antegrade wire track may be mandatory, so that further salvage procedures such as thrombus aspiration or embolisation for haemostasis can be performed.

\section{IPSILATERAL CHANNEL COLLATERAL RETROGRADE CTO PCI WITH SINGLE GUIDING CATHETER}

Although we usually recommend a ping-pong guiding catheter technique when dealing with ipsilateral collateral retrograde CTO PCI, there are instances where we might use the retrograde approach through a single GC. If access is impossible, or if we begin with a single GC for an antegrade CTO PCI but decide to try a difficult retrograde channel with a low success rate, we might forego a second femoral puncture on anticoagulation for a brief try in the channel through the single GC. Sometimes we face an easy retrograde approach with ipsilateral collateral and we might attempt it through a single GC. In all these cases, the rendezvous technique is essential as we cannot trap the retrograde wire with balloon anchoring in the GC, and we often cannot push the retrograde $\mathrm{MC}$ through the CTO lesion. This remains a valid reason for the rendezvous technique.

\section{Tips and tricks for rendezvous techniques POSITION OF THE RENDEZVOUS}

The easiest position to achieve rendezvous is at the level of the secondary bend of the antegrade GC. The tips of the MC and wire will both lean against the outer curvature of the catheter, and therefore it will be easy to advance the wire into the $\mathrm{MC}$ at this level (Figure 4A).

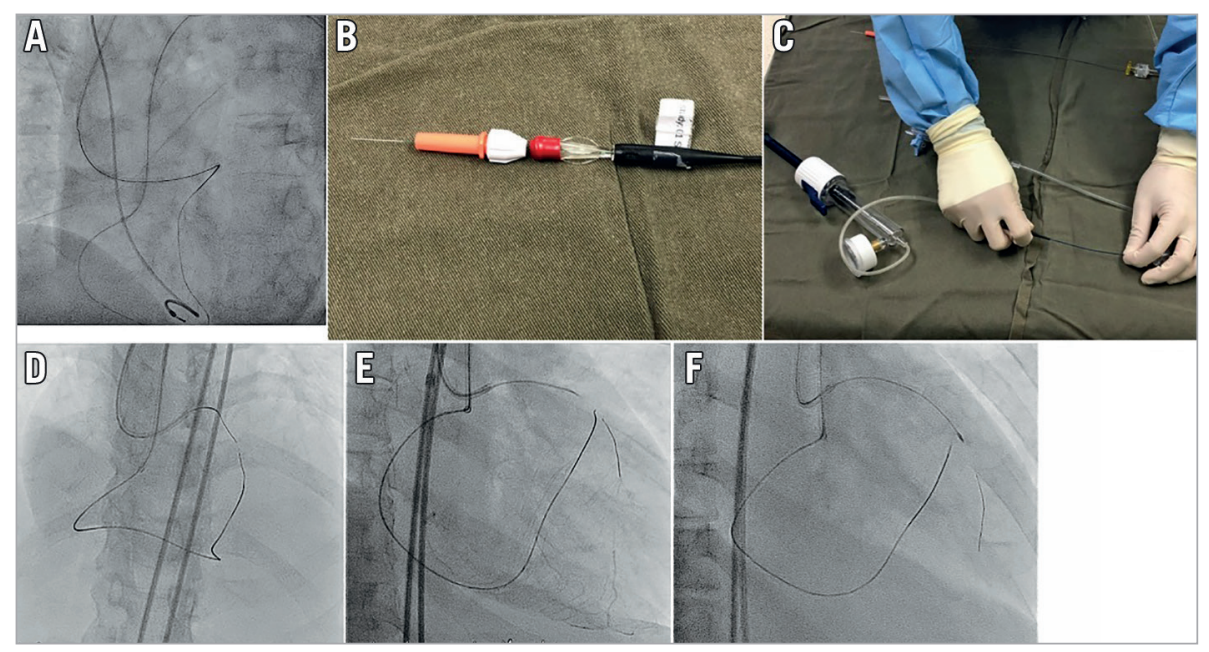

Figure 4. Rendezvous and tip-in. A) Best bending position of the antegrade guide for tip-in. B) Locking torque device on retrograde side of wire after tip-in. C) Applying negative pressure to improve wire tracking. D) Antegrade RotaWire tracking retrograde microcatheter tip-in technique. E) Successful antegrade RotaWire passage to distal true lumen. F) 1.25 mm burr to CTO lesion. 


\section{RAPID SWITCHING TO ANTEGRADE}

After tip-in, the retrograde wire should be advanced as far as possible into the antegrade MC. A torque device is then locked firmly on the remaining end of the wire against the retrograde $\mathrm{MC}$ hub (Figure 4B) to prevent loss of wire control. The antegrade MC now can be pushed, meeting the retrograde MC tip, continuously across the CTO segment ${ }^{29}$.

\section{WHEN MC CANNOT CROSS THE CTO BODY AFTER RENDEZVOUS}

If the indication for the rendezvous technique is the inability to pass the retrograde $\mathrm{MC}$ through the CTO body, then we need to maximise the antegrade MC's penetrative power to cross the CTO. In these cases we should start with the maximal back-up and most powerful MC. When we suspect that the MC cannot cross the CTO body, we should change to a strong back-up support antegrade GC, engage a guide extension catheter, or use an anchor balloon in a side branch. A special penetrative MC, such as Turnpike Gold (Teleflex) or Turnpike Spiral (Teleflex) may also help. Applying continuous negative suction to the antegrade penetrative MC (Figure 4C) may pull the retrograde wire inside indirectly, and increase the penetrative force of the antegrade MC.

\section{ROTAWIRE EXCHANGE TECHNIQUE}

If all the techniques in the section above are tried and the antegrade MC still cannot be crossed, and the remaining distance between the antegrade and retrograde MCs is short, we can attempt advancing an antegrade rotablator wire into the retrograde $\mathrm{MC}$. The retrograde wire is withdrawn to the tip of the antegrade $\mathrm{MC}$, then a RotaWire $^{\mathrm{TM}}$ (Boston Scientific) is placed into the antegrade MC until its tip is in contact with the retrograde wire tip. The retrograde wire is then gradually pulled back a short distance, with the antegrade RotaWire advanced to follow closely (Figure 4D). This will allow the RotaWire to track the void of the removed retrograde wire, and achieve wire exchange. Once the RotaWire is across the CTO body and has entered the distal vessel (Figure 4E), subsequent rotational atherectomy can be carried out to complete the CTO intervention (Figure 4F).

\section{COMPARISON OF METHODS TO OVERCOME A CTO SEGMENT WHERE MC PASSAGE IS DIFFICULT}

There are several options to overcome this problem when a retrograde wire has crossed the $\mathrm{CTO}$ but the $\mathrm{MC}$ cannot cross. Continuing aggressive pushing on the retrograde $\mathrm{MC}$ can lead to rupture of the retrograde channel or retrograde $\mathrm{MC}$ tip deformation, both resulting in the loss of retrograde access. Exchanging the retrograde MC for a small balloon to dilate the lesion is also associated with risks of channel injury. Giving up the original wire position and performing retrograde knuckle wiring across CTO segment via the subintimal space, or conversion to traditional $\mathrm{CART}^{20}$, are both time-consuming and do not guarantee success. External cap crush carries the risk of proximal vessel injury, haematoma extension, and perforation. Therefore, a well-executed tip-in type rendezvous followed by a dedicated penetrative antegrade MC is probably the most reasonable option $^{30}$. Antegrade RotaWire change is also relatively simple and with low risk.

\section{Conclusions}

The technical success of CTO PCI is usually very high after successful rev-CART. However, the operator may still be unable to establish antegrade wire access, resulting in final failure. This gap is not well covered in previous literature.

We, the APCTO club, have provided this review and recommendations for methods to achieve antegrade wire access after successful rev-CART. We have addressed every failure mode in detail, covering different options and their risks. Our recommendations focus upon safety first, and then ease of use. We hope this work will help all retrograde operators to further improve their procedures.

\section{Conflict of interest statement}

S.A. Harding has received speaking and consultancy fees from Boston Scientific, Medtronic, Bio-Excel, and Asahi Intecc. E.B. Wu has received proctoring fees from Boston Scientific, Abbott Vascular, and research grants from Orbus Neich and Asahi Intecc. S. Lo has received speaking and proctoring honoraria from Bio-Excel. S.T. Lim has received research grant/travel support or speaker honoraria from Orbus Neich, Asahi Intecc, Terumo, Biosensors, Biotronik, Abbott Vascular, Aluimedica, Boston Scientific, and Keneka. E. Tsuchikane is a consultant for Boston Scientific, Asahi Intecc, Kaneka, and Nipro. The other authors have no conflicts of interest to declare.

\section{References}

1. Surmely JF, Tsuchikane E, Katoh O, Nashida Y, Nakayama M, Nakamura S, Oida A, Hattori E, Suzuki T. New concept for CTO recanalization using controlled antegrade and retrograde subintimal tracking: the CART technique. J Invasive Cardiol. 2006;18:334-8.

2. Surmely JF, Katoh O, Tsuchikane E, Nasu K, Suzuki T. Coronary septal collaterals as an access for the retrograde approach in the percutaneous treatment of coronary chronic total occlusions. Catheter Cardiovasc Interv. 2007;69:826-32.

3. Tsuchikane E, Katoh O, Kimura M, Nasu K, Kinoshita Y, Suzuki T. The first clinical experience with a novel catheter for collateral channel tracking in retrograde approach for chronic coronary total occlusions. JACC Cardiovasc Interv. 2010;3:165-71.

4. Matsuno S, Tsuchikane E, Harding SA, Wu EB, Kao HL, Brilakis ES, Masahyekhi K, Werner GS. Overview and proposed terminology for the reverse controlled antegrade and retrograde tracking (reverse CART) techniques. EuroIntervention. 2018;14: 94-101.

5. Galassi AR, Sianos G, Werner GS, Escaned J, Tomasello SD, Boukhris M, Castaing M, Büttner JH, Bufe A, Kalnins A, Spratt JC, Garbo R, Hildick-Smith D, Elhadad S, Gagnor A, Lauer B, Bryniarski L, Christiansen EH, Thuesen L, Meyer-Geßner M, Goktekin O, Carlino M, Louvard Y, Lefèvre T, Lismanis A, Gelev VL, 
Serra A, Marzà F, Di Mario C, Reifart N; Euro CTO Club. Retrograde Recanalization of Chronic Total Occlusions in Europe: Procedural, In-Hospital, and Long-Term Outcomes From the Multicenter ERCTO Registry. J Am Coll Cardiol. 2015;65:2388-400.

6. Karmpaliotis D, Michael TT, Brilakis ES, Papayannis AC, Tran DL, Kirkland BL, Lembo N, Kalynych A, Carlson H, Banerjee S, Lombardi W, Kandzari DE. Retrograde coronary chronic total occlusion revascularization: procedural and in-hospital outcomes from a multicenter registry in the United States. JACC Cardiovasc Interv. 2012;5:1273-9.

7. Michael TT, Mogabgab O, Alomar M, Kotsia A, Christopoulos G, Rangan BV, Abdullah S, Grodin J, Banerjee S, Brilakis ES. Longterm outcomes of successful chronic total occlusion percutaneous coronary interventions using the antegrade and retrograde approach. J Interv Cardiol. 2014;27:465-71.

8. Karmpaliotis D, Karatasakis A, Alaswad K, Jaffer FA, Yeh RW, Wyman RM, Lombardi WL, Grantham JA, Kandzari DE, Lembo NJ, Doing A, Patel M, Bahadorani JN, Moses JW, Kirtane AJ, Parikh M, Ali ZA, Kalra S, Nguyen-Trong PK, Danek BA, Karacsonyi J, Rangan BV, Roesle MK, Thompson CA, Banerjee S, Brilakis ES. Outcomes With the Use of the Retrograde Approach for Coronary Chronic Total Occlusion Interventions in a Contemporary Multicenter US Registry. Circ Cardiovasc Interv. 2016;9:e003434.

9. Rinfret S, Joyal D, Nguyen CM, Bagur R, Hui W, Leung R, Larose E, Love MP, Mansour S. Retrograde recanalization of chronic total occlusions from the transradial approach; early Canadian experience. Catheter Cardiovasc Interv. 2011;78:366-74.

10. Huang Z, Ma D, Zhang B, Folson AA, Lin J, Wu K, Liao H, Zhong Z. Epicardial collateral channel for retrograded recanalization of chronic total occlusion percutaneous coronary intervention: predictors of failure and procedural outcome. J Interv Cardiol. 2018;31:23-30.

11. Lee CK, Chen YH, Lin MS, Yeh CF, Hung CS, Kao HL, Huang CC. Retrograde Approach is as Effective and Safe as Antegrade Approach in Contemporary Percutaneous Coronary Intervention for Chronic Total Occlusion: A Taiwan Single-Center Registry Study. Acta Cardiol Sin. 2017;33:20-7.

12. Maeremans J, Walsh S, Knaapen P, Spratt JC, Avran A, Hanratty CG, Faurie B, Agostoni P, Bressollette E, Kayaert P, Bagnall AJ, Egred M, Smith D, Chase A, McEntegart MB, Smith WH, Harcombe A, Kelly P, Irving J, Smith EJ, Strange JW, Dens J. The hybrid algorithm for treating chronic total occlusions in Europe. The RECHARGE registry. J Am Coll Cardiol. 2016;68: 1958-70.

13. Wu EB, Tsuchikane E, Lei G, Harding S, Lo S, Lim ST, Chen JY, Lee SW, Qian J, Kao HL, Yan BPY. Retrograde Versus Antegrade Approach for Coronary Chronic Total Occlusion in an AlgorithmDriven Contemporary Asia-Pacific Multicenter Registry: Comparison of Outcomes. Heart Lung Circ. 2020;29:894-903.

14. Thompson CA. The hybrid approach for percutaneous revascularization of coronary chronic total occlusions. Interv Cardiol Clin. 2012;1:349-53.
15. Brilakis ES, Grantham JA, Rinfret S, Wyman RM, Burke MN, Karmpaliotis D, Lembo N, Pershad A, Kandzari DE, Buller CE, DeMartini T, Lombardi WL, Thompson CA. A percutaneous treatment algorithm for crossing coronary chronic total occlusions. $\mathrm{J} \mathrm{Am}$ Coll Cardiol Interv. 2012;5:367-79.

16. Joyal D, Thompson CA, Grantham JA, Buller CE, Rinfret S. Retrograde technique for recanalization of chronic total occlusions: a step-by-step approach. JACC Cardiovasc Interv. 2012;5:1-11.

17. Brilakis ES, Grantham JA, Thompson CA, DeMartini TJ, Prasad A, Sandhu GS, Banerjee S, Lombardi WL. The retrograde approach to coronary artery chronic total occlusions: a practical approach. Catheter Cardiovasc Interv. 2012;79:3-19.

18. Wu EB, Chan WW, Yu CM. Retrograde chronic total occlusion intervention: tips and tricks. Catheter Cardiovasc Interv. 2008; 72:806-14

19. Harding SA, Wu EB, Lo S, Lim ST, Ge L, Chen JY, Qian J, Lee SW, Kao HL, Tsuchikane E. A new algorithm for crossing chronic total occlusions from the Asia Pacific Chronic Total Occlusion Club. J Am Coll Cardiol Interv. 2017;10:2135-43.

20. Wu EB, Tsuchikane E, Lo S, Lim ST, Ge L, Chen JY, Qian J, Lee SW, Harding SA, Kao HL. Retrograde algorithm for chronic total occlusion from the Asia Pacific Chronic Total Occlusion club. AsiaIntervention. 2018;4:98-107.

21. Wu EB, Tsuchikane E, Lo S, Lim ST, Ge L, Chen JY, Qian J, Lee SW, Kao HL, Harding SA. Chronic Total Occlusion Wiring: A State-of-the-Art Guide From the Asia Pacific Chronic Total Occlusion Club. Heart Lung Circ. 2019;28:1490-500.

22. Mozid AM, Davies JR, Spratt JC. The utility of a guideliner catheter in retrograde percutaneous coronary intervention of a chronic total occlusion with reverse CART - the "capture" technique. Catheter Cardiovasc Interv. 2014;83:929-32.

23. Otsuka Y, Kataoka Y, Fukuoka T. Snaring the retrograde wire in the aortic root for chronic total occlusion after Bentall operation in a patient with Bechet's disease. J Invasive Cardiol. 2009;21: E137-40

24. Fang HY, Lee WC, Fang CY, Wu CJ. Application of a snare technique in retrograde chronic total occlusion percutaneous coronary intervention- a step by step practical approach and an observational study. Medicine. 2016;95:e5129.

25. Wu EB, Tsuchikane E. The inherent catastrophic traps in retrograde CTO PCI. Catheter Cardiovasc Interv. 2018;91:1101-9.

26. Yokoi K, Sumitsuji S, Kaneda H, Siegrist PT, Okayama K, Ide S, Mizote I, Kumada M, Kuroda T, Tachibana K, Sakata Y, Nanto S. A novel homemade snare, safe, economical and sizeadjustable. EuroIntervention. 2015;10:1307-10.

27. Kim MH, Yu LH, Mitsudo K. A new retrograde wiring technique for chronic total occlusion. Catheter Cardiovasc Interv. 2010;75:117-9.

28. Muramatsu T, Tsukahara R, Ito Y. "Rendezvous in coronary" technique with the retrograde approach for chronic total occlusion. J Invasive Cardiol. 2010;22:E179-82.

29. Funatsu A, Kobayashi T, Nakamura S. Use of the kissing microcatheter technique to exchange a retrograde wire for an 
antegrade wire in the retrograde approach to intervention in chronic total occlusion. J Invasive Cardiol. 2010;22(5):E74-7.

30. Vo MN, Ravandi A, Brilakis ES. "Tip-in” technique for retrograde chronic total occlusion revascularization. J Invasive Cardiol. 2015;27:E62-4.

31. Vo MN, Ravandi A, Grantham JA. Subintimal space plaque modification for "Balloon-uncrossable" chronic total occlusions. J Invasive Cardiol. 2014;26:E133-6.

\section{Supplementary data}

Moving image 1. Retrograde wire path. Pushing the retrograde wire and noting its position, and then manipulating the ipsilateral ping-pong guide to the position of the retrograde wire path.

Moving image 2. Successful wiring of the antegrade guiding catheter.

The supplementary data are published online at: www.asiaintervention.org 